\title{
METHOD OF TESTING AND CORRECTING SIGNAL AMPLIFIERS' TRANSFER FUNCTION USING PRONY ANALYSIS
}

\author{
Jarosław Zygarlicki ${ }^{1)}$, Janusz Mroczka ${ }^{2)}$ \\ 1) Opole University of Technology, Institute of Power Engineering, Prószkowska 76, 45-758 Opole, Poland (هj.zygarlicki@po.opole.pl, \\ +48774000 547) \\ 2) Wroclaw University of Technology, Chair of Electronic and Photonic Metrology, Bolesława Prusa 53/55, 50-317 Wrocław, Poland \\ (janusz.mroczka@pwr.wroc.pl, +48 713206232 )
}

\begin{abstract}
This article presents a way of analyzing the transfer function of electronic signal amplifiers. It also describes the possibility of using signal precorrection which improves the parasitic harmonics in the THD (Total Harmonic Distortion) of the amplified signal by correcting linearity of the tested amplifier's transfer function. The proposed method of analyzing and presenting the transfer function allows to diagnose the causes of generating parasitic harmonics, what makes it a useful tool when designing low distortion amplifier systems, such as e.g. amplifiers in measurement systems. The presented THD correction can be used in e.g. amplifier systems that cooperate with arbitrary generators.
\end{abstract}

Keywords: Prony method, signal processing, harmonics, measurements.

(C) 2012 Polish Academy of Sciences. All rights reserved

\section{Introduction}

Besides their signal amplifying function, electronic amplifiers are the source of different distortions that deteriorate the parameters of the output signal in relation to the input signal. There are different kinds of amplifier distortions. Analysis of these distortions enables diagnosis of the amplifier system, elimination of distortions by modifying the amplifier system or their correction by using e.g. signal processing methods.

In general, two groups of distortions in amplifier systems can be distinguished. The first group is constituted by linear distortions. The transfer function of the device generating linear distortions has a straight line. Linear distortions cause phase shifts of signal sinusoidal components and modify of amplification in the frequency of the amplified component. Linear distortions do not depend on the amplitude of the input signal. Distortions of this kind are presented by means of amplitude and phase/frequency characteristics and are relatively simple to measure and correct [1].

Nonlinear distortions form the second group of distortions. Such distortion brings the generation of sinusoidal components that are not inherent in the input signal. Distortions of this kind result from nonlinearity of amplifier transfer function and depend on the amplitude of the input signal.

There are several ways of testing nonlinear distortions. The transfer function can be tested directly step by step, and the quantitative parameters of distortions can be calculated on the basis of obtained results. However, due to insignificant distortion in modern amplifiers this method is highly imprecise. The second way is to excite the amplifier system with a wellknown signal and to analyze the amplifier output components that are absent in the test signal. This, however, generates the numerical parameter describing the distortion, which does not allow more precise diagnosis of the distortions origin. 
This article focuses on nonlinear distortion that is difficult to diagnose and eliminate. It proposes a new method - a tool for testing and analyzing distortions of this kind. The presented solution is characterised by high precision, it enables amplifier diagnosis and the analysis of the distortion origins, as well as in special amplifier applications - digital correction of these distortions.

The article is divided into six sections. Introduction is the first section of the article. Section two describes the genesis of the proposed method. Section three presents the proposed algorithms and the way of conducting measurements. Section four contains test results. Section five covers the analysis and summary of the tests. The final sixth section presents conclusions.

\section{Genesis of the article}

Nonlinear distortions result from nonlinearity of the system transfer function. Several nonlinearity types for amplifier transfer function can be distinguished [2]. The shape of nonlinearity reveals the cause of distortion. Fig. 1 presents the diagram of typical distortions.
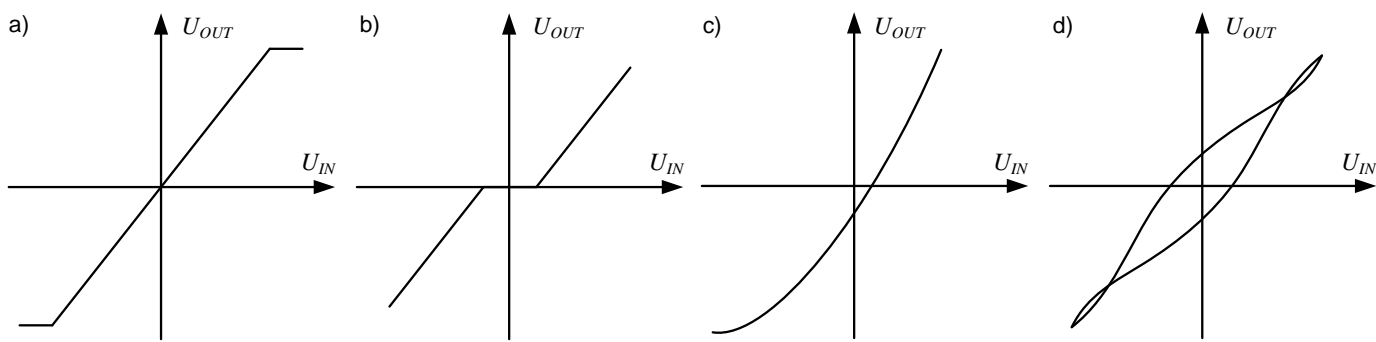

Fig. 1. Types of transfer function of signal amplifiers.

Fig. 1a presents distortions that are a result of exceeding the amplitude range of the amplified signals. This can occur in effect of feeding the signal with too high amplitude or of incorrect amplitude ranges of individual sections of a multistage amplifier.

Fig. $1 \mathrm{~b}$ reveals the nature of the amplifier in which the positive and negative parts of the signal are amplified separately. Distortion within the beginning of the coordinate system suggests insufficient calibration of the moment of activating individual sections of this kind of amplifier.

Fig. 1c presents characteristics of the amplifier whose transfer function is not symmetrical to the beginning of the coordinate system and greater distortion is visible in one of the characteristics quarters. Distortion of such transfer function is caused by the improper setting of the operating point of the amplifying element, mainly in class A amplifiers. Amplifiers offering such characteristics generate even harmonics in the amplified signal.

Fig. 1d shows different characteristics for the fragments of the signal with momentary amplitude increase and a different one for the signals with momentary amplitude decrease. Such characteristics reveals significant phase shifts induced by the amplifier in the signal frequency. Characteristics of this kind cannot be obtained by applying a step by step testing method.

From the parameters used for quantitative description of nonlinear distortion, such as: THD+N (Total Harmonic Distortion plus Noise), IMD (Intermodulation Distortion) or TIM (Transient Intermodulation Distortion) [3], the most popular parameter is the THD (Total Harmonic Distortion) [4], defined as the ratio of the amplitude of the higher harmonics of the signal to the fundamental harmonic amplitude value, and measured for a sinusoidal signal: 


$$
\mathrm{THD}=\frac{\sqrt{\sum_{k=2}^{M} U_{h k}^{2}}}{U_{h 1}},
$$

where: $M$ is the number of harmonics for which the factor is measured, $U_{h k}$ is the amplitude of the $k$-th harmonic for $k=1,2, \ldots, M, U_{h 1}$ is the amplitude of the fundamental harmonic.

The THD measurement can be carried out in the form of Fourier's analysis $[5,6]$ of the input signal at excitation by a sinusoidal signal.

THD can also be measured indirectly, when the shape of the amplifier's transfer function is known. One of the methods is the approximation method or the so-called five ordinates method - Fig. 2.

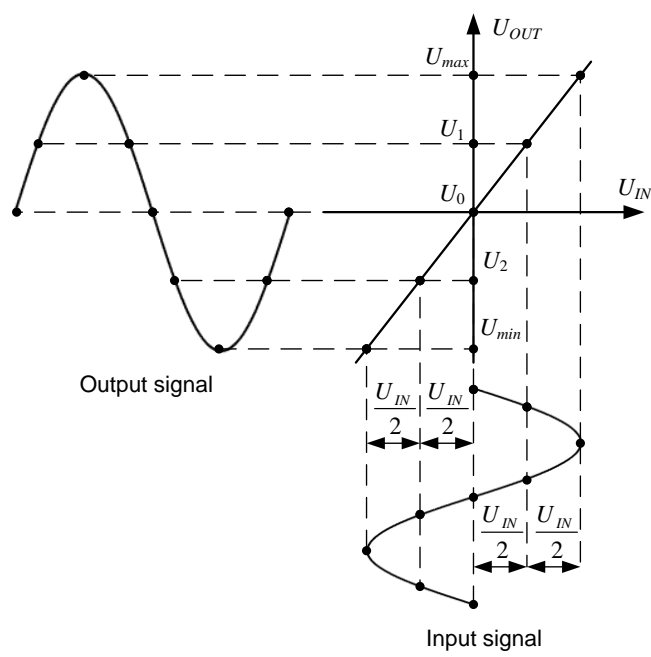

Fig. 2. Five ordinates method for THD marking.

This method can be described by means of the following dependencies:

$$
\begin{aligned}
& U_{h 1}=\frac{U_{\max }-U_{\text {min }}+U_{1}-U_{2}}{3}, \\
& U_{h 2}=\frac{U_{\text {max }}+U_{\text {min }}-2 U_{0}}{4}, \\
& U_{h 3}=\frac{U_{\text {max }}-U_{\text {min }}-2\left(U_{1}-U_{2}\right)}{6}, \\
& U_{h 4}=\frac{U_{\text {max }}+U_{\min }-4\left(U_{1}+U_{2}\right)+6 U_{0}}{12},
\end{aligned}
$$

where symbols in the patterns are explained in Fig. 2. The equations (2-5) are valid for a sinusoidal signal at the input.

As can be deducted from the patterns, the shape of the transfer function is clearly connected with both the THD parameter and the content of individual harmonics.

On the basis of the information about harmonics generated at the output of the studied system excited with a sine wave, the authors conclude that it is possible to perform an operation that is opposite to the one presented above, i.e. to reconstruct the transfer function of the system.

The reconstructed characteristics obtained in this way will not be able to clearly reveal the 
distortion induced by the harmonics, as modern amplifiers offer very good linearity. Therefore, the authors developed the idea of amplifying all harmonics (excluding the first one) proportionally in the analyzed system response in an artificial way, prior to reconstructing its transfer function. By their transfer function, the amplified distortions should allow to clearly analyze and diagnose the origins of harmonics generated in the tested amplifier system.

\section{Method description and methodology of study}

A simplified presentation of the proposed method of analyzing and visualizing the transfer function of signal amplifiers can be seen in Fig. 3.

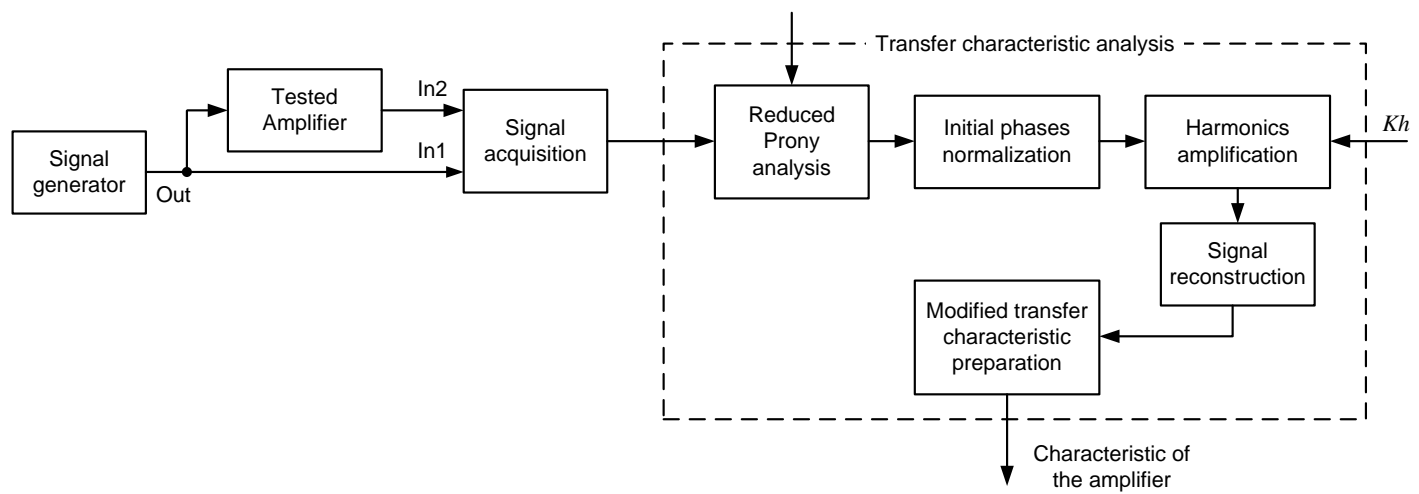

Fig. 3. Method of analysis of the tested system's transfer function.

The authors' original method assumes the following operations:

- calculation of the test signal and sending it to the output of the measuring card,

- acquisition of measuring card signals, In1 and In2 inputs,

- calculation of initial phases and amplitudes of harmonics of the recorded signals by means of the reduced Prony method,

- recalculation of the fundamental harmonic phase in relation to the time delay for the recorded signals,

- reconstruction of signals of In1 and In2 inputs on the basis of calculated harmonic parameters with time vectors corrected by phase delays of signal fundamental components,

- calculation of initial phases and amplitudes of harmonics of the reconstructed signals by means of the reduced Prony method,

- recalculation of the amplitudes of harmonics (excluding the fundamental harmonic) by the set amplification value $K h$,

- reconstruction of signals on the basis of modified harmonic parameters - amplified harmonic amplitudes,

- defining the transfer function (the abscissae make modified reconstructed transient voltage values of input $\mathrm{In} 1$ and the ordinates make modified reconstructed transient voltage values of input In2),

- defining the average transfer function.

The influence of the amplifier noise is neutralized in the presented method by computing the average of 10 measured characteristics. More details of the reduced Prony method used is presented in publication [7].

The proposed way of correcting the transfer function of signal amplifiers is presented in Fig. 4. The above method can be described by the following stages: 
a) Switches in Fig. 4 in default position,

- specifying the transfer function of the amplifier for a set signal, for $K h=1$, in accordance with the diagram in Fig. 3,

b) Switches in Fig. 4 in changed position in relation to the default position,

- multiplying time samples of the regenerated set signal by correction points specified on the basis of the transfer function,

- sending the corrected signal to the system input after amplification.

The tests presented below were conducted with the use of a PC computer equipped with measuring card NI PCI-6120, in the configuration presented in Fig. 5. The analogue output of the measuring card is connected to the input of the tested amplifier and, additionally, to the analogue input of the first In1, which facilitates measurement of signal phase shifts in the amplifier system. The amplifier output is connected to the resistor serving as load and to the second input In2 of the measuring card.

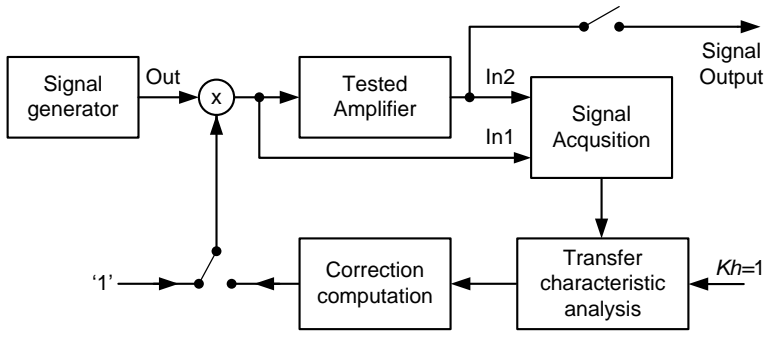

Fig. 4. Method of generating a corrected amplified signal.

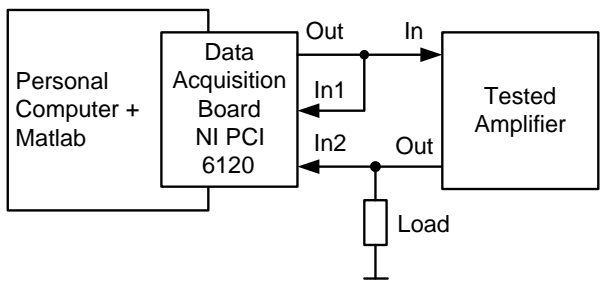

Fig. 5. Measurement system for testing the amplifier transfer function.

The measuring card used in the tests has two C/A 16-bit converters offering a sampling frequency of up to $4 \mathrm{MHz}$ and input signal amplitude up to $10 \mathrm{~V}$, as well as four 16-bit $\mathrm{A} / \mathrm{C}$ converters offering a sampling frequency of up to $800 \mathrm{kHz}$ and a voltage range up to $\pm 42 \mathrm{~V}$. The card also features the option of storing $64 \mathrm{M}$ of samples in the built-in RAM memory, an antialiasing filter and the simultaneous sampling option. The Matlab computational environment was used for generation, acquisition and processing of the test signal. The frequency of the generated test signal was $1 \mathrm{kHz}$. Testing of amplifier system characteristics was carried out using Prony's method [8-12], that was applied for analysis of successive harmonics generated in the tested amplifier system for 1 to 20 components.

\section{Results}

Measurements were carried out for three different amplifiers:

- amplifier $I$ - system built on the self-contained operational power amplifier (OPA 541) in typical application,

- amplifier II - power amplifier system operating in class B, final phase built on discrete bipolar junction transistors,

- amplifier III - power amplifier system operating in class A with an output transformer.

The results of testing the characteristics and possibilities of correcting it using the authors' original methods are presented in Fig. 6 - 21. Figs. 22 to 24 show the analyses of the measurement system alone (excluding the tested amplifier). Figs. 6, 10, 14, 18 shows ranges of the input amplitudes of the tested amplifiers. 


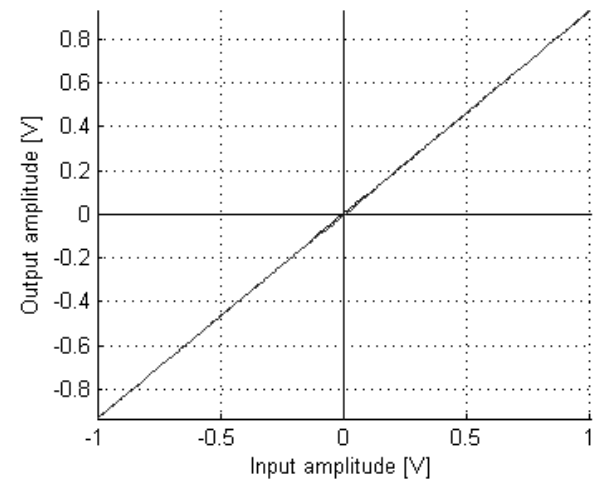

Fig. 6. Transfer function of amplifier I.

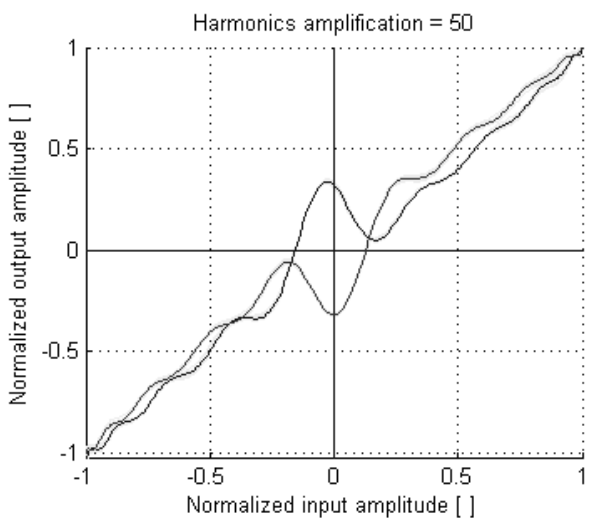

Fig. 7. Transfer function of amplifier I with amplified harmonics.

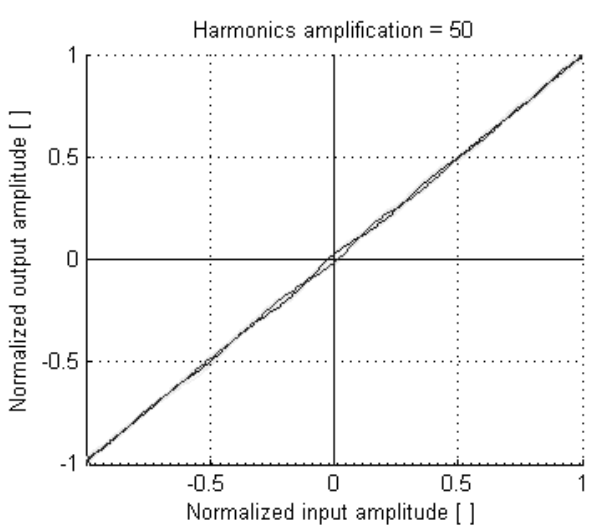

Fig. 8. Corrected transfer function of amplifier I with amplified harmonics.

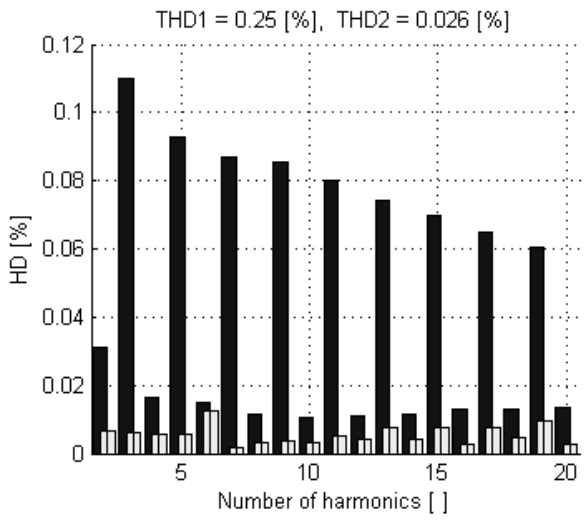

Fig. 9. Comparison of harmonic distortion of amplifier I without correction (THD1, dark color graph) and with correction (THD2, light color).

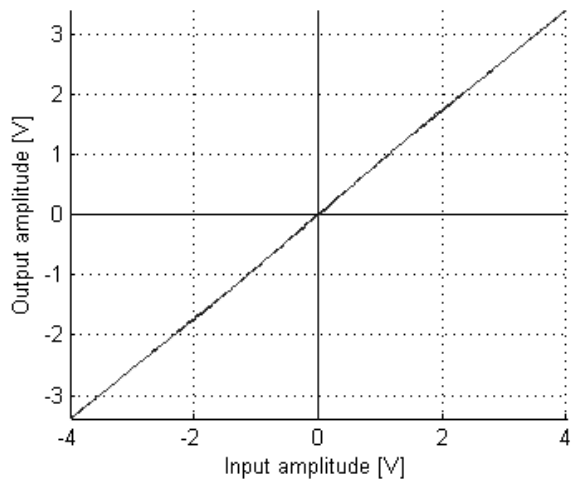

Fig. 10. Transfer function of amplifier I.

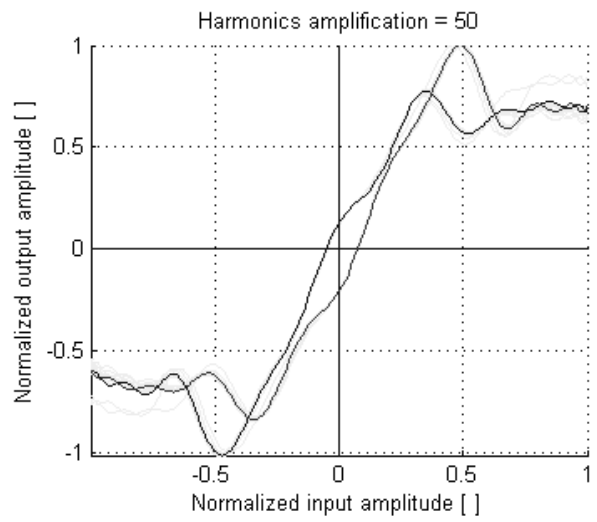

Fig. 11. Transfer function of amplifier I with amplified harmonics.

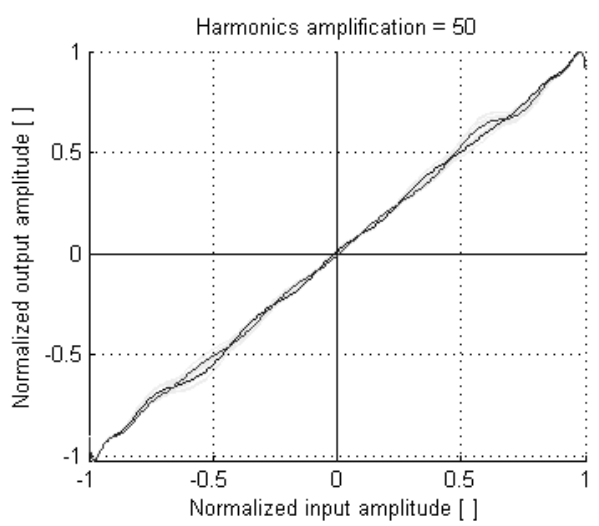

Fig. 12. Corrected transfer function of amplifier I with amplified harmonics.

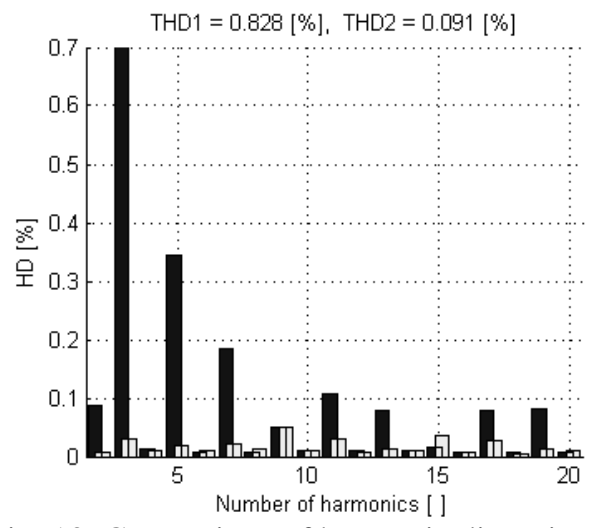

Fig. 13. Comparison of harmonic distortion of amplifier I without correction (THD1, dark color graph) and with correction (THD2, light color). 


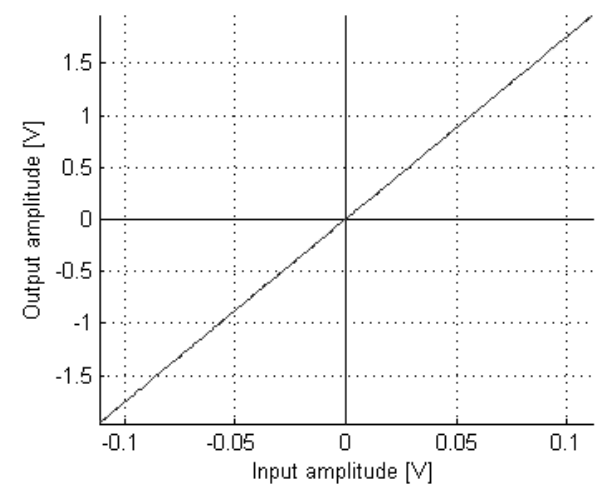

Fig. 14. Transfer function of amplifier II.

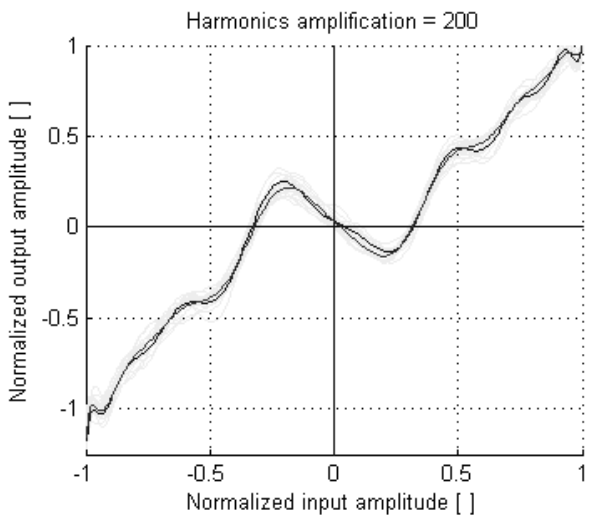

Fig. 15. Transfer function of amplifier II with amplified harmonics.

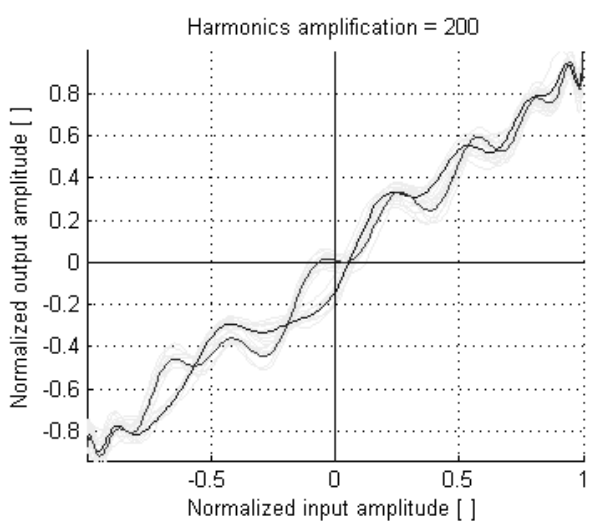

Fig. 16. Corrected transfer function of amplifier II with amplified harmonics.

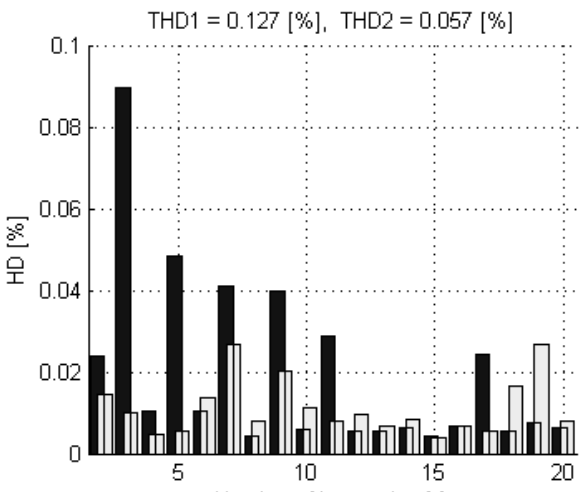

Number of harmonics [

Fig. 17. Comparison of harmonic distortion of amplifier II without correction (THD1, dark color graph) and with correction (THD2, light color).

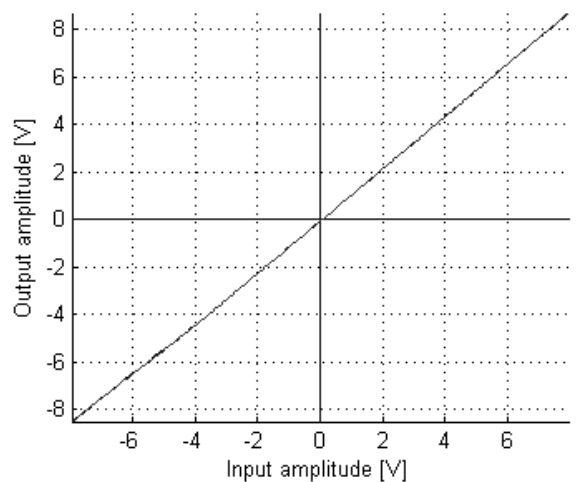

Fig. 18. Transfer function of amplifier III.

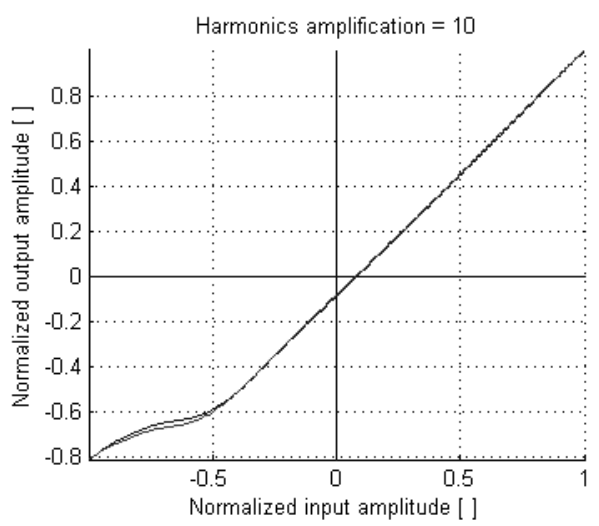

Fig. 19. Transfer function of amplifier III with amplified harmonics.

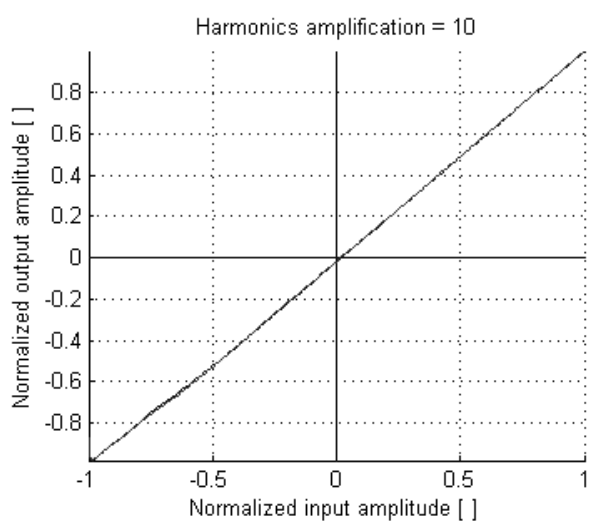

Fig. 20. Corrected transfer function of amplifier III with amplified harmonics.

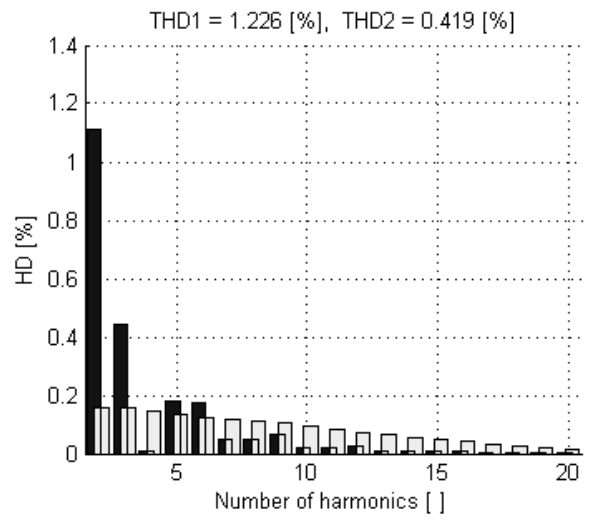

Fig. 21. Comparison of harmonic distortion of amplifier III without correction (THD1, dark color graph) and with correction (THD2, light color). 


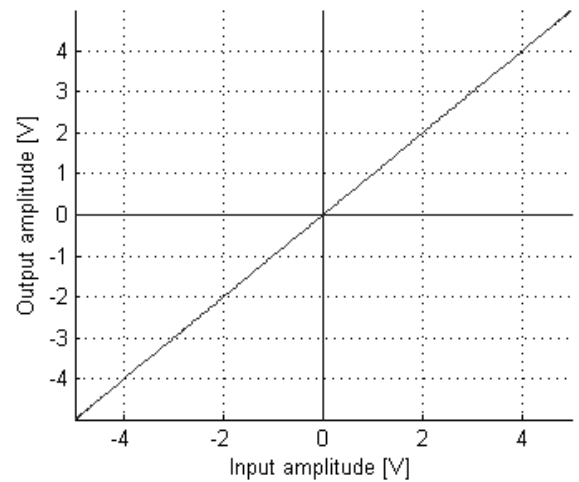

Fig. 22. Transfer function of the measurement system.

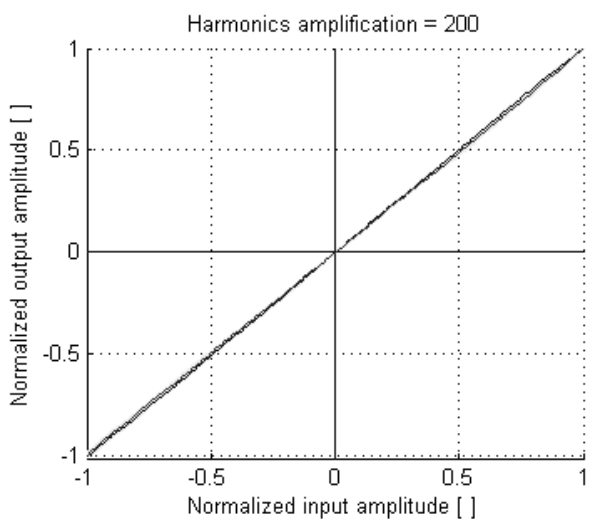

Fig. 23. Transfer function of the measurement system with amplified harmonics.

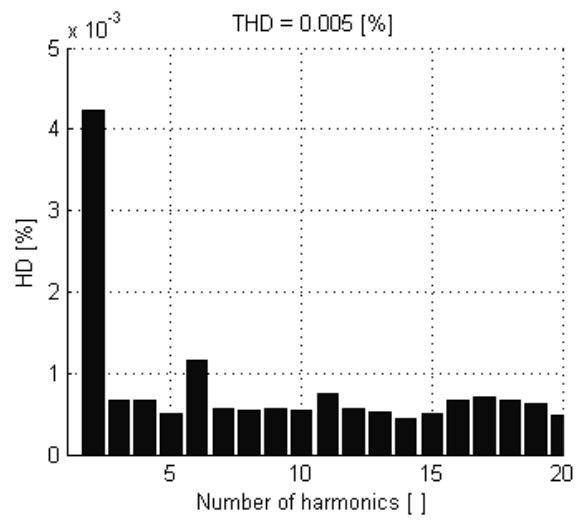

Fig. 24. Level of harmonic distortion in the measurement system.

\section{Summary}

Figs. 6, 10, 14 and 18 show the transfer function of the tested amplifiers without amplification of parasitic harmonics. Besides defining the voltage amplification of given amplifiers, specifying other parameters is not possible. Nonlinear distortions are almost indiscernible and their presence can only be recognized with the use of diagrams presenting the content of harmonics in the output signal. Figs. 7, 11, 15 and 19 reveal amplitudenormalised characteristics with amplified harmonics. The amplification value was selected on the basis of successive increase up to obtaining clear distortion of individual characteristics. For amplifier I harmonics amplification of 50 was specified, for amplifier II 200, and for amplifier III 10. The value of the specified harmonics amplification is connected with the quality of a given amplifier-introduced harmonics distortion (THD). What is visible are specific distortions induced by given amplifier systems that are impossible to read and analyze using a traditional transfer function or diagram with harmonics.

Figs. 8, 12, 16 and 20 show characteristics of signals corrected so as to eliminate the distortion induced by amplifier systems. Just like the characteristics in Figs. 7, 11, 15 and 19, these diagrams present the normalized form of transfer function also with amplified harmonics in order to facilitate their comparison prior to correction. After correction, the linearity of the characteristics is significantly better. The algorithm of correction is shown in Fig. 4 and described in Section 3.

The effect of the correction and harmonic content in the tested amplifiers before and after correction are shown in Figs. 9, 13, 17 and 21. In each of the tested cases, improvement 
(reduction) in the content of harmonics was achieved, with the value of $9.6 \mathrm{x}$ and $9.1 \mathrm{x}$ for amplifier I, $2.2 \mathrm{x}$ for amplifier II and $2.9 \mathrm{x}$ for amplifier III.

On the basis of the waveform of each characteristic it is possible to diagnose the cause of the occurrence of harmonics in given amplifier systems.

In case of amplifier I (Fig. 7) and amplifier II (Fig. 15) the greatest distortion occurs within the pass through the beginning of the normalized coordinate system. The recorded shape of the characteristics suggests that the construction of the class B amplifiers should allow for separate amplification of the signals with negative and positive amplitudes. Distortions within that area prove insufficient calibration of moments (amplitudes) of activation of individual amplifier sections.

Moreover, the hysteresis of the amplifier I characteristics, in the form of different characteristics for the fragments of signals with increasing momentary voltage amplitude and for signals with decreasing momentary amplitude, is an evidence of significant phase shifts induced by amplifier I as a function of signal component frequency, which can be of great importance for certain amplifier applications. The phase shifts introduced by the amplifier may be caused by parasitic capacities.

In relation to amplifier I, the amplifier II is characterised by smaller phase distortions (the characteristic in Fig. 15 does not show hysteresis) and the harmonics are amplified 200 times, which proves greater linearity of the system than in amplifier I for which $K h=50$ was adopted. The THD of amplifier II is low, so harmonics amplification must have been set to a greater value to enable observation of nonlinearity of the transfer function.

Figs. 10 to 13 present the results for amplifier I with the increased amplitude of the input signal (Fig. 10). The calculated characteristics (Fig. 11) show significant distortions at the end of the charts, which suggests that the amplitude range of the input signal was exceeded. The above-mentioned phenomenon does not occur at the unloaded output of the circuit. We also know that the self-contained power amplifier OPA541, a component of amplifier I, is supplied with a symmetric voltage of $+/-15 \mathrm{~V}$. We can thus conclude that the current efficiency of the power supply used in the amplifier I is insufficient to guarantee minimum nonlinear distortions, or the distortions were induced by the current protection of the amplifier outputs.

The characteristics of the amplifier III were presented in Figs. 18 - 21. This is a class-A amplifier with an output transformer. The type of the amplifier is also confirmed by the graph in Fig. 19. The characteristics show clear distortions already at $K h=10$, which proves significant nonlinear distortion of the amplifier system. The characteristics are not symmetrical to the beginning of the coordinate system. The problem with disturbances within the beginning of the ordinate system does not exist, either, which is beneficial when amplifying signals with high dynamic level and components with relatively high and relatively low amplitudes. The greatest distortion occurs at one end of the characteristics, due to the use of an output transformer whose core is made of a material offering certain nonlinear magnetization characteristics and due to the fact that the amplifier system is not subject to global feedback loop that could eliminate the observed effect to a certain extent.

To evaluate the influence of the measurement system on the results of the analyses, we tested the system with the amplifier substituted by a short section of screened wire. The tests conducted for the system modified in this way are presented in Figs. 22 - 24. As can be seen in the presented characteristics, the distortions induced by the measurement system are negligibly small as compared to the distortions induced by the tested amplifiers.

The frequency of the generated test signal was $1 \mathrm{kHz}$, what is typical value. The measured transfer function for this selected signal frequency, can be transferred to the complete frequency range of the amplifier. Some differences in transfer function appearance may occur for higher frequencies, because of parasitic capacitances in the amplifier structure. 


\section{Conclusions}

This article presented a way of analyzing the transfer function of electronic signal amplifiers. By using the proposed method, we analyzed three examples of amplifier circuits and on the basis of obtained results we diagnosed the causes of generating nonlinear distortions in the amplified signal by the tested systems.

The study revealed that the proposed solution can be a useful tool in designing lowdistortion amplifier systems, e.g. amplifiers in measurement systems, which would provide better diagnostics based on the characteristics of the content of individual harmonics in the input signal alone.

The article also describes the possibility of using signal precorrection that improves the parasitic harmonics in the THD of the amplified signal. The presented THD correction can be used in amplifier systems cooperating with e.g. arbitrary generators.

\section{References}

[1] Zygarlicki, J., Mroczka, J. (2011). Practical use of Reduced Prony's method - investigations of voltage input circuit for power monitoring system. Electrical Review, 199-203.

[2] Vamsi, Paidi, Shouxuan, Xie, Robert, Coffie, et al. (2003). High Linearity and High Efficiency of ClassB Power Amplifiers in GaN HEMT Technology. IEEE Trans. on Microwave Theory and Techniques, 51(2), 643-653.

[3] Thalayasingam, K., Heuermann, H. (2009). Novel vector non-linear measurement system for intermodulation measurements. In Proc. Microwave Conference, EuMC 2009, 926-929.

[4] Palumbo, G., Pennisi, S. (2003). High-frequency harmonic distortion in feedback amplifiers: analysis and applications. IEEE Transactions on Circuits and Systems, 50(3), 328-340.

[5] Borkowski, J. (2011). Minimization of maximum errors in universal approximation of the unit circle by a polygon. Metrol. Meas. Syst., 18(3), 391-402.

[6] Borkowski, J., Mroczka, J. (2010). LIDFT method with classic data windows and zero padding in multifrequency signal analysis. Measurement (London), 43(10), 1595-1602.

[7] Zygarlicki, J., Zygarlicka, M., Mroczka, J., Latawiec, K. (2010). A reduced Prony's method in power quality analysis - parameters selection. IEEE Transactions on Power Delivery, 25(2), 979-986.

[8] Zygarlicki, J., Mroczka, J. (2011). Short time algorithm of power waveforms fundamental harmonic estimations with Prony's methods use. Metrol. Meas. Syst., 18(3), 33-38.

[9] Zygarlicki, J., Mroczka, J. (2012). Variable-frequency Prony method in the analysis of electrical power quality. Metrol. Meas. Syst., 19(1), 39-48.

[10] Rulian, Zhang, Chengyong, Zhao (2010). Prony analysis of electrical transient characteristics of AC system during HVDC commutation failure. In Proc. IPEC, 2010 Conference Proceedings, 807-812.

[11] Feilat, E.A. (2006). Prony analysis technique for estimation of the mean curve of lightning impulses. IEEE Trans. Power Del., 21(4), 2088-2090.

[12] Lobos, T., Leonowicz, Z., Rezmer, J., Schegner P. (2006). High-resolution spectrum-estimation methods for signal analysis in power systems. IEEE Trans. Instrum. Meas., 55(1), 219-225. 